International Journal of Number Theory

Vol. 6, No. 8 (2010) 1945-1950

(C) World Scientific Publishing Company

DOI: $10.1142 / \mathrm{S} 1793042110003885$

\title{
AUTHOR INDEX (Volume 6)
}

\begin{abstract}
A
Agashe, A., Squareness in the special $L$-value and special L-values of twists

Ahmadi, O., Shparlinski, I. E. \& Voloch, J. F., Multiplicative order of Gauss periods
\end{abstract}

Alaca, A., Alaca, Ş. \& Williams, K. S., Fourteen octonary quadratic forms

Alaca, A., Alaca, Ş. \&

Williams, K. S., Sextenary quadratic forms and an identity of Klein and Fricke

Alaca, S.., see Alaca, A.

Alaca, Ş. see Alaca, A.

B

Baczkowski, D., Filaseta, M., Luca, F. \& Trifonov, O., On values of $d(n !) / m !, \phi(n !) / m !$ and $\sigma(n !) / m$ !

Balazard, M. \& de Roton, A., Sur un critère de Báez-Duarte pour l'hypothèse de Riemann

Baoulina, I., On the number of solutions to certain diagonal equations over finite fields

Barman, R. \& Saikia, A., Iwasawa $\lambda$-invariants and $\Gamma$-transforms of $p$-adic measures on $\mathbb{Z}_{p}^{n}$

Beck, M. \& Halloran, M., Finite trigonometric character sums via discrete Fourier analysis

Belabas, K. \& Fouvry, É.,
5 (2010) 1091

$4(2010) 877$

1 (2010) 37

1 (2010) 169

1 (2010) 37

1 (2010) 169

6 (2010) 1199

4 (2010) 883

1 (2010) 1

8 (2010) 1819

1 (2010) 51
Discriminants cubiques et progressions arithmétiques

7 (2010) 1491

Bennett, M. A., Ellenberg, J. S. \& Ng, N. C., The

Diophantine equation $A^{4}+2^{\delta} B^{2}=C^{n}$

2 (2010) 311

Bettin, S., The second moment of the Riemann zeta function with unbounded shifts

Booher, J., Etropolski, A. \& Hittson, A., Evaluations of cubic twisted Kloosterman sheaf sums

6 (2010) 1349

Borwein, J. M. \& Chan, O.-Y., Duality in tails of multiple-zeta values

Bostan, A., Salvy, B. \& Tran, K., Generating functions of Chebyshev-like polynomials

Bouallègue, K., Echi, O. \& Pinch, R. G. E., Korselt numbers and sets

Boylan, M., Arithmetic properties of certain level one mock modular forms

8 (2010) 1933

3 (2010) 501

7 (2010) 1659

2 (2010) 257

Bremner, A. \& Spearman, B. K., Cyclic sextic trinomials $x^{6}+A x+B$

Brown, J., Special values of $L$-functions on $\mathrm{GSp}_{4} \times \mathrm{GL}_{2}$ and the non-vanishing of Selmer groups

8 (2010) 1901

Brown, J., The first negative Hecke eigenvalue of genus 2 Siegel cuspforms with level $N \geq 1$
1 (2010) 185

1 (2010) 161 
Bugeaud, Y. \& Mignotte, M., Polynomial root separation

3 (2010) 587

C

Cai, T., see Xia, B.

Cai, Y., Lagrange's four squares theorem with variables of special type

Caranay, P. C. \& Scheidler, R., An efficient seventh power residue symbol algorithm

Chan, H.-C., Ramanujan's cubic continued fraction and an analog of his "Most Beautiful Identity"

Chan, H.-C., Ramanujan's cubic continued fraction and Ramanujan type congruences for a certain partition function

Chan, H. H., Cooper, S. \& Sica, F., Congruences satisfied by Apéry-like numbers

Chan, O.-Y., see Borwein, J. M.

Chao, K. F. \& Plymen, R., $A$ new bound for the smallest $x$ with $\pi(x)>\operatorname{li}(x)$

Chu, W. \& Zhang, W., Bilateral Bailey lemma and false theta functions

Coons, M., The transcendence of series related to Stern's diatomic sequence

Cooper, S., see Chan, H. H.

Copil, V. \& Panaitopol, L., On the ratio of consecutive primes

D

David, C. \& Urroz, J. J., Square-free discriminants of Frobenius rings

de Roton, A., see

Balazard, M.

Del Corso, I. \& Dvornicich, R., Non-invariance of the index in wildly ramified extensions
4 (2010) 849

8 (2010) 1801

8 (2010) 1831

3 (2010) 673

4 (2010) 819

$1(2010) 89$

3 (2010) 501

3 (2010) 681

3 (2010) 515

1 (2010) 211

1 (2010) 89

1 (2010) 203

6 (2010) 1391

4 (2010) 883

8 (2010) 1855
Dewar, M. \& Richter, O. K., Ramanujan congruences for Siegel modular forms

7 (2010) 1677

Dujella, A. \& Jurasić, A., On the size of sets in a polynomial variant of a problem of Diophantus

Dvornicich, R., see Del Corso, I.

7 (2010) 1449

8 (2010) 1855

E

Echi, O., see

Bouallègue, K.

Ehlen, S., Twisted Borcherds products on Hilbert modular surfaces and the regularized theta lift

7 (2010) 1473

Ellenberg, J. S., see

Bennett, M. A.

Etropolski, A., see Booher, J.

2 (2010) 311

6 (2010) 1349

\section{F}

Fehm, A. \& Petersen, S., On the rank of abelian varieties over ample fields

3 (2010) 579

Feigon, B. \& Whitehouse, D., Exact averages of central values of triple product L-functions

7 (2010) 1609

Filaseta, M., see Baczkowski, D.

6 (2010) 1199

7 (2010) 1491

Fouvry, E., see Belabas, K.

Frisch, S., see

Vaserstein, L.

6 (2010) 1261

G

Ganguly, S., On the dimension of the space of cusp forms of octahedral type

4 (2010) 767

Garvan, F. G., Congruences for Andrews' smallest parts partition function and new congruences for Dyson's rank

2 (2010) 281

Gottesman, R. \& Tang, K., Quadratic recurrences with a positive density of prime divisors

$5(2010) 1027$

$\mathbf{H}$

Halloran, M., see Beck, M. 1 (2010) 51 
Hambleton, S. \&

Scharaschkin, V.,

Quadratic reciprocity via resultants

Han, J. S., Kim, H. S. \&

Neggers, J., The

Fibonacci-norm of a

positive integer:

Observations and

conjectures

Hasegawa, T., On asymptotically optimal towers over quadratic fields related to Gauss hypergeometric functions

Hassen, A. \& Nguyen, H. D., Hypergeometric zeta functions

Haynes, A. K., Numerators of differences of nonconsecutive Farey fractions

Hittson, A., see Booher, J.

Hoelscher, J. L., Ray class groups of quadratic and cyclotomic fields

Hoshi, A. \& Miyake, K., On the field intersection problem of solvable quintic generic polynomials

I

Ih, S.-I. \& Tucker, T. J., A finiteness property for preperiodic points of Chebyshev polynomials

J

Jahangiri, M., Generators of arithmetic quaternion groups and a

Diophantine problem

Jouhet, F. \& Mosaki, E., Irrationalité aux entiers impairs positifs d'un q-analogue de la fonction zêta de Riemann

Jurasić, A., see Dujella, A.

K

Kable, A. C., $A n$ arithmetical invariant of orbits of affine actions and its application to similarity classes of quadratic spaces
6 (2010) 1413

2 (2010) 371

5 (2010) 989

1 (2010) 99

3 (2010) 655

6 (2010) 1349

5 (2010) 1169

5 (2010) 1047

5 (2010) 1011

6 (2010) 1311

7 (2010) 1449

6 (2010) 1215
Kane, B., Representations of integers by ternary quadratic forms

1 (2010) 127

Kaneko, M. \& Ohno, Y., On a kind of duality of multiple zeta-star values

Kang, S.-Y. \& Kim, C. H., Arithmetic properties of traces of singular moduli on congruence subgroups

Katayama, K., Generalized gamma functions with characters

8 (2010) 1927

8 (2010) 1755

Katre, S. A., see Tanti, J.

Kelmer, D., Distribution of twisted Kloosterman sums modulo prime powers

2 (2010) 271

Kida, M., Rikuna, Y. \& Sato, A., Classifying Brumer's quintic polynomials by weak Mordell-Weil groups

Kim, B., Combinatorial proofs of certain identities involving partial theta functions

Kim, C. H., see Kang, S.-Y.

7 (2010) 1625

6 (2010) 1329

Kim, H. S., see Han, J. S.

Kim, S., A bijective proof of the quintuple product identity

$2(2010) 247$

Kleinbock, D., Margulis, G. \& Wang, J., Metric Diophantine approximation for systems of linear forms via dynamics

5 (2010) 1139

Knopp, M. \& Raji, W., Eichler cohomology for generalized modular forms II

3 (2010) 691

2 (2010) 449

8 (2010) 1755

2 (2010) 371

Kohnen, W., A short note on Fourier coefficients of half-integral weight modular forms

6 (2010) 1255

Krieg, A., Theta series over the Hurwitz quaternions

1 (2010) 25

L

Lagarias, J. C., Cyclic systems of simultaneous congruences 
Laishram, S., On a conjecture on Ramanujan primes

Lalín, M. N., On a conjecture by Boyd

Le, D., Manber, S. \& Shah, S., On p-adic properties of twisted traces of singular moduli

Lebacque, P., On Tsfasman-Vlădut invariants of infinite global fields

Lee, K. S. E., On the sum of a prime and $a$ Fibonacci number Lev, V. F., Muzychuk, M. E. \& Pinchasi, R. Additive bases in abelian groups

Li, W., see Zhao, Y.

Lozano-Robledo, Á. \& Lundell, B., Bounds for the torsion of elliptic curves over extensions with bounded ramification

Luca, F., see Baczkowski, D.

Lucht, L. G., A survey of Ramanujan expansions

Lundell, B., see Lozano-Robledo, Á.

\section{M}

Maier, H. \&

Sankaranarayanan, A., Exponential sums over primes in residue classes

Mammo, B., On the density of discriminants of abelian extensions of $a$ number field

Manber, S., see Le, D.

Margulis, G., see Kleinbock, D.

Matomäki, K., A note on smooth numbers in short intervals

McCarthy, D., ${ }_{3} F_{2}$ hypergeometric series and periods of elliptic curves

Mignotte, M., see Bugeaud, Y.
3 (2010) 461

8 (2010) 1869

3 (2010) 705

3 (2010) 625

6 (2010) 1419

7 (2010) 1669

4 (2010) 799

2 (2010) 437

6 (2010) 1293

6 (2010) 1199

8 (2010) 1785

6 (2010) 1293

4 (2010) 905

6 (2010) 1273

3 (2010) 625

5 (2010) 1139

5 (2010) 1113

3 (2010) 587
Miller, A. \& Pixton, A., Arithmetic traces of non-holomorphic modular invariants

Miyake, K., see Hoshi, A.

Mosaki, E., see Jouhet, F.

Munshi, R., Density of positive rank fibers in elliptic fibrations, II

Muzychuk, M. E., see Lev, V. F.

1 (2010) 69

5 (2010) 1047

5 (2010) 959

1 (2010) 15

4 (2010) 799

N

Nebe, G. \& Venkov, B., Low-dimensional strongly perfect lattices. III: Dual strongly perfect lattices of dimension 14

2 (2010) 387

2 (2010) 371

Neggers, J., see Han, J. S.

Ng, N. C., see Bennett, M. A.

2 (2010) 311

Nguyen, H. D., see Hassen, A.

1 (2010) 99

0

Ohno, Y., see Kaneko, M.

8 (2010) 1927

$\mathbf{P}$

Panaitopol, L., see Copil, V.

1 (2010) 203

Patkowski, A. E., On curious generating functions for values of L-functions

7 (2010) 1531

Petersen, S., see Fehm, A.

Pickett, E. J., Construction of self-dual integral normal bases in abelian extensions of finite and local fields

7 (2010) 1565

Pinch, R. G. E., see Bouallègue, $\mathrm{K}$.

2 (2010) 257

4 (2010) 799

1 (2010) 69

Pixton, A., see Miller, A.

Plymen, R., see Chao, K. F.

3 (2010) 681

$\mathbf{R}$

Raji, W., see Knopp, M.

5 (2010) 1083

Richter, O. K., see Dewar, M.

Rikuna, Y., see Kida, M.

Robertson, L., Monogeneity in cyclotomic fields

7 (2010) 1677

3 (2010) 691

7 (2010) 1589

Rowell, M., A new exploration of the Lebesgue identity 
Roy, D., Small value estimates for the additive group

Rühl, K.-T., Annihilating ideals of quadratic forms over local and global fields

3 (2010) 603

$\mathrm{S}$

Saikia, A., see Barman, R.

Sairaiji, F., Formal groups of Jacobian varieties of hyperelliptic curves

Sakkalis, T., see Vaserstein, L.

Salvy, B., see Bostan, A.

Samuels, C. L., The finiteness of computing the ultrametric Mahler measure

Sankaranarayanan, A., see Maier, H.

Sato, A., see Kida, M.

Sbeity, F. \& Sodaïgui, B., Classes de Steinitz d'extensions non abéliennes à groupe de Galois d'ordre 16 ou extraspécial d'ordre 32 et problème de plongement

Scharaschkin, V., see Hambleton, S.

Scheidler, R., see Caranay, P. C.

Scheidler, R., see $\mathrm{Wu}, \mathrm{Q}$.

Schwab, E. D., Generalized arithmetical functions of three variables

Shah, S., see Le, D.

Shemanske, T., Treneer, S. \& Walling, L.,

Constructing

simultaneous Hecke

eigenforms

Shparlinski, I. E., see Ahmadi, O.

Sica, F., see Chan, H. H.

Sinick, J., Ramanujan congruences for a class of eta quotients

Sodaïgui, B., see Sbeity, F.

Soudères, I., Motivic double shuffle

8 (2010) 1819

7 (2010) 1701

6 (2010) 1261

7 (2010) 1659

8 (2010) 1731

4 (2010) 905

3 (2010) 691

8 (2010) 1769

6 (2010) 1413

8 (2010) 1831

7 (2010) 1541

7 (2010) 1689

3 (2010) 625

5 (2010) 1117

4 (2010) 877

1 (2010) 89

4 (2010) 835

8 (2010) 1769

2 (2010) 339

Spearman, B. K., see Bremner, A.

1 (2010) 161

\section{T}

Tang, K., see Gottesman, R.

5 (2010) 1027

Tanti, J. \& Katre, S. A., Euler's criterion for septic nonresidues

6 (2010) 1329

Templier, N., On asymptotic values of canonical quadratic L-functions 8 (2010) 1717 7 (2010) 1659

Tran, K., see Bostan, A.

Treneer, S., see Shemanske, $\mathrm{T}$.

Trifonov, O., see Baczkowski, D.

5 (2010) 1117

6 (2010) 1199

Tucker, T. J., see Ih, S.-I.

5 (2010) 1011

\section{U}

Ulas, M., Variations on higher twists of pairs of elliptic curves

5 (2010) 1183

Urroz, J. J., see David, C.

6 (2010) 1391

\section{V}

Vaserstein, L., Sakkalis, T. \& Frisch, S., Polynomial parametrization of Pythagorean tuples

Venkov, B., see Nebe, G.

Verrill, H. A., Congruences related to modular forms

6 (2010) 1261

2 (2010) 387

6 (2010) 1367

Viada, E., Lower bounds for the normalized height and non-dense subsets of subvarieties of abelian varieties

3 (2010) 471

Vienney, M., A new construction of $p$-adic Rankin convolutions in the case of positive slope

Villa-Salvador, G., An elementary proof of the conductor-discriminant formula

Volkov, M., Abelian surfaces with supersingular good reduction and non-semisimple Tate module

4 (2010) 811

Voloch, J. F., see Ahmadi, O.

4 (2010) 877
5 (2010) 1191

8 (2010) 1875
Vulakh, L. Ya., Diophantine approximation in 
imaginary quadratic fields

Vulakh, L. Ya., Hermitian points in Markov spectra

Vulakh, L. Ya., Minima of binary indefinite Hermitian forms

\section{W}

Walling, L., see Shemanske, T.

Wang, J., see Kleinbock, D.

Whitehouse, D., see Feigon, B.

Williams, G., Unimodular integer circulants associated with trinomials

Williams, K. S., see Alaca, A.

Williams, K. S., see Alaca, A.

\begin{tabular}{|c|c|}
\hline $4(2010)$ & 731 \\
\hline $4(2010)$ & 713 \\
\hline $2(2010)$ & 411 \\
\hline $5(2010)$ & 1117 \\
\hline 5 (2010) & 1139 \\
\hline 7 (2010) & 1609 \\
\hline $4(2010)$ & 869 \\
\hline 1 (2010) & 37 \\
\hline 1 (2010) & 169 \\
\hline
\end{tabular}

Witno, A., On elite primes of period four

3 (2010) 667

Wu, Q. \& Scheidler, R.,

The ramification groups and different of a compositum of Artin-Schreier extensions

7 (2010) 1541

$\mathrm{X}$

Xia, B. \& Cai, T., Bernoulli numbers and congruences for harmonic sums

4 (2010) 849

Z

Zhang, W., see Chu, W.

Zhang, X., see Zhao, Y.

Zhao, Y., Li, W. \& Zhang, $\mathrm{X}$., Effective determination of prime decompositions of cubic function fields
3 (2010) 515

2 (2010) 437

$2(2010) 437$ 